> Mycobacterium abscessus est une bactérie non tuberculeuse, environnementale, à croissance rapide, qui est responsable d'infections pulmonaires sévères, notamment chez les patients atteints de mucoviscidose. Le traitement actuel combine l'utilisation d'une $\beta$-lactamine et d'un aminoglycoside associés à un macrolide. Cette bactérie est polyrésistante à la plupart des antibiotiques utilisés en clinique. Les mécanismes de résistance, innés ou acquis, qu'elle a développés, conduisent fréquemment à des échecs thérapeutiques, ce qui limite considérablement les moyens de lutte disponibles pour le clinicien. Une compréhension globale des mécanismes de résistance de cette bactérie s'avère ainsi nécessaire pour contrer les infections pulmonaires qu'elle provoque.<

Mycobacterium abscessus a été isolée pour la première fois en 1950, par Morris Moore et John B Frerichs, chez une patiente de 63 ans ayant consulté pour divers troubles dont un abcès situé au niveau du genou, résultant d'un traumatisme survenu 48 ans plus tôt [1]. Cette mycobactérie non tuberculeuse (MNT) à croissance rapide est classée dans le phylum des Actinobactéries (Figure 1A). M. abscessus regroupe en fait trois sous-espèces bactériennes: $M$. abscessus subsp. abscessus, M. abscessus subsp. bolletii et M. abscessus subsp. massiliense. Celles-là diffèrent par leur profil génétique mais aussi par leur susceptibilité à certains antibiotiques [2]. M. abscessus est un pathogène opportuniste intracellulaire (Figure lB) qui est souvent assimilé à d'autres mycobactéries. Confondue avec ces autres espèces, sa prévalence propre pourrait ainsi être largement sous-estimée. Son mode de transmission reste encore largement incompris. Néanmoins, une étude réalisée en 2016 suggère une transmission interhumaine possible chez les patients atteints de mucoviscidose [3]. M. abscessus est responsable de manifestations cliniques très étendues. Outre des infections cutanées, osseuses, disséminées et, voire, dans de rares cas, lorsque la barrière

Vignette (Photo ㄷ Wassim Daher).

\section{Mycobacterium abscessus, un modèle de résistance aux différentes classes d'antibiotiques}

\author{
Morgane Illouz ${ }^{1, *}$, Matthéo Alcaraz ${ }^{1, *}$, \\ Françoise Roquet-Banères ${ }^{1}$, Laurent Kremer ${ }^{1,2}$
}

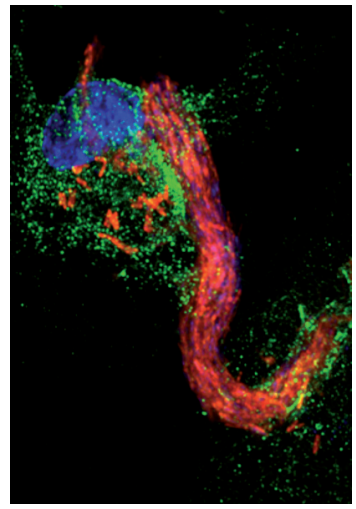

${ }^{1}$ CNRS UMR 9004,

Institut de recherche en infectiologie de Montpellier (IRIM), Université de Montpellier, 1919 route de Mende, 34293, Montpellier, France. ${ }^{2}$ Inserm, IRIM, 34293 Montpellier, France.

${ }^{*}$ Contribution équivalente laurent.kremer@irim.cnrs.fr

hémato-encéphalique est altérée, d'atteintes du système nerveux central, M. abscessus est responsable d'infections pulmonaires sévères, en particulier chez les patients souffrant de troubles pulmonaires comme la mucoviscidose [4]. On estime en effet que 10 à $20 \%$ de ces patients, qui présentent une susceptibilité accrue aux infections bactériennes, sont infectés par une MNT (principalement M. abscessus et Mycobacterium avium). L'infection par M. abscessus est l'une des infections bactériennes les plus difficiles à traiter : elle est en effet multi-résistante aux antibiotiques, et le taux d'échec des traitements atteint près de $60 \%$ [5]. Sa place de pathogène émergent s'explique par son incidence, qui peut surpasser, dans certains pays industrialisés, celle de Mycobacterium tuberculosis, l'agent étiologique de la tuberculose [4]. Le séquençage complet du génome de $M$. abscessus a révélé la présence de plusieurs gènes codant des facteurs de virulence spécifiques de M. tuberculosis ainsi que de nombreux facteurs de virulence non mycobactériens, caractéristiques d'autres pathogènes souvent associés à la mucoviscidose, tels que Pseudomonas aeruginosa $[6,7]$. La grande plasticité du génome de M. abscessus et les nombreux mécanismes de résistance aux antibiotiques que déploie cette bactérie témoignent de sa capacité d'adaptation à son environnement.

\section{M. abscessus, une menace croissante pour les patients atteints de mucoviscidose?}

La mucoviscidose est une maladie génétique héréditaire causée par une mutation du gène codant la protéine CFTR (cystic fibrosis transmembrane regulator) [8] $(\rightarrow$ ).

$(\rightarrow)$ Voir la Synthèse de C. Ferec, $m / s$ $n^{\circ}$ 6-7, juin-juillet 2021, page 618 

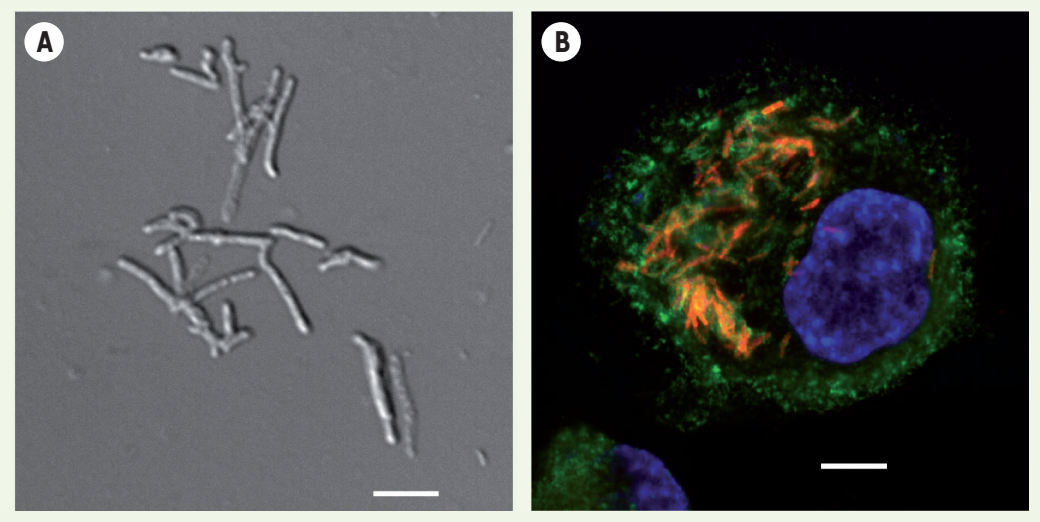

Figure 1. M. abscessus, un bacille intracellulaire. A. Image de microscopie en contraste de phase de M. abscessus. L'échelle représente $5 \mu \mathrm{m}$. B. Image d'immunofluorescence d'un macrophage infecté par M. abscessus exprimant TdTomato (rouge). Les macrophages sont détectés grâce à un anticorps anti-CD63 (vert) et les noyaux sont marqués au DAPI (bleu). L'échelle représente $10 \mu \mathrm{m}$.

Cette maladie autosomique récessive affecte, en particulier, les fonctions digestives et respiratoires. La protéine CFTR est en effet une protéine transmembranaire qui joue un rôle de canal ionique localisé au pôle apical des cellules épithéliales pulmonaires, permettant l'échange d'ions chlorure et sodium au travers de la membrane des cellules [9]. Des mutations du gène CFTR entraînent un dysfonctionnement à l'origine d'une mauvaise hydratation du mucus bronchique, qui, de ce fait, devient de plus en plus épais et visqueux, favorisant ainsi l'établissement d'infections polymicrobiennes chroniques. M. abscessus représente, avec $M$. avium, la MNT la plus fréquemment retrouvée dans les expectorations de patients atteints de mucoviscidose, pouvant induire un déclin rapide de leurs fonctions pulmonaires [10]. Le dysfonctionnement de CFTR réduit, entre autre, l'activité bactéricide des macrophages en raison d'une diminution de leur production de radicaux oxydants en réponse aux infections [11]. À l'incapacité du système immunitaire inné à contrôler efficacement les infections bactériennes, s'ajoute la difficulté, pour le clinicien, à traiter ces infections et à éradiquer le bacille responsable [12]. La présence de M. abscessus peut aussi représenter une contre-indication à la greffe pulmonaire, un recours souvent nécessaire chez les patients atteints de mucoviscidose.

\section{L'antibiothérapie comme traitement des infections à M. abscessus}

La majorité des molécules utilisées en clinique dans le traitement contre M. abscessus ont un effet bactériostatique : elles ne font que stopper la prolifération bactérienne, sans induire la mort des bactéries. Ces molécules ne montrent également que peu ou pas d'activité vis-à-vis des biofilms, constructions complexes formées de colonies bactériennes, ce qui pourrait expliquer la relative inefficacité des régimes thérapeutiques actuellement appliqués [13]. Pendant plusieurs années, les recommandations thérapeutiques ont été fondées sur une trithérapie, associant une $\beta$-lactamine (imipénème ou céfoxitine), un aminoglycoside (amikacine), administrés par voie parentérale pendant 12 mois, et un macrolide (clarithromycine ou azithromycine), par voie orale. Afin d'optimiser cette thérapie chez les personnes atteintes de mucoviscidose, un consortium regroupant I'US Cystic Fibrosis Foundation et l'European Cystic Fibrosis Society, préconise maintenant une première phase de thérapie dite intensive (durant au moins un mois), suivie d'une phase de prolongation (de 12 mois) (Figure 2). Cette stratégie prend en compte le profil de résistance aux antibiotiques de la souche à l'origine de l'infection. II est donc important de différencier les isolats cliniques de M. abscessus qui sont sensibles ou résistants aux antibiotiques, dont les macrolides. L'action combinée de plusieurs antibiotiques permet de cibler différentes voies métaboliques utilisées par la bactérie afin de l'éradiquer (Figure 3) [14].

\section{Les classes d'antibiotiques}

Les $\beta$-lactamines regroupent une large classe d'antibiotiques (dont les pénicillines, les céphalosporines, les carbapénèmes). Elles agissent en inhibant la synthèse d'un composant essentiel de la paroi des mycobactéries, le peptidoglycane, formé d'une épine dorsale alternant des chaînons $\mathrm{N}$-acétyl glucosamine - acide $\mathrm{N}$-acétyl muramique, et de chaînes tétrapeptidiques (L-Alaninyl-D-isoGlutaminyl-meso-diaminopimelylD-Alanine) liées à l'acide muramique. Lors de l'étape finale de la synthèse de cet hétéropolymère, des liaisons covalentes se forment entre les chaînes latérales des peptides monomères qui le composent, produisant la macromolécule qui englobera la membrane plasmique. La réticulation du peptidoglycane est assurée par des transpeptidases bactériennes qui appartiennent à la famille des protéines liant la pénicilline (PBP), les D,D-transpeptidases (DDT) et les L,D-transpeptidases (LDT), qui catalysent respectivement des liaisons de type $4 \rightarrow 3$ et $3 \rightarrow 3$ entre ces chaines peptidiques latérales [15]. Les $\beta$-lactamines agissent sur ces transpeptidases en entrant en compétition, par mimétisme, avec le substrat de ces enzymes, bloquant ainsi la biosynthèse du peptidoglycane. Les céphalosporines et les 


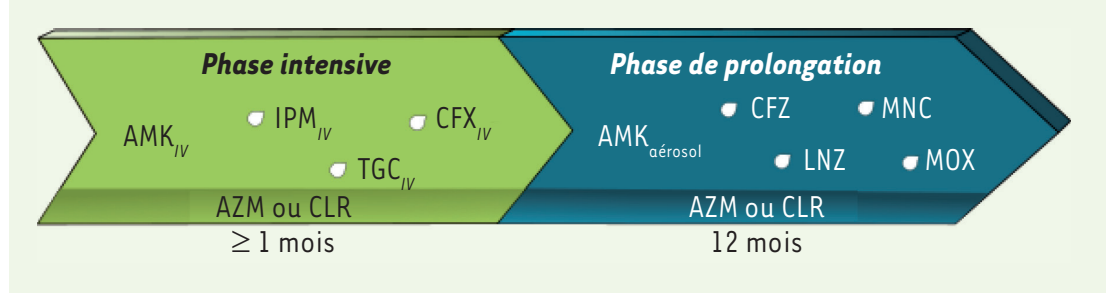

Figure 2. Régime thérapeutique dans le cadre d'infections à M. abscessus. La phase intensive, d'une durée pouvant excéder un mois, comporte une dose quotidienne d'amikacine injectée par voie intraveineuse $\left(A M K_{I V}\right)$, d'un antibiotique choisi parmi l'imipénème (IPM), la tigécycline (TGC) ou la céfoxitine (CFX), ainsi qu'un macrolide (azithromycine (AZM)

ou clarithromycine (CLR)). La phase de prolongation, d'une durée d'environ 12 mois, comprend un macrolide, de l'AMK en aérosol ainsi que 2 à 3 antibiotiques choisis parmi la clofazimine (CFZ), le linézolide (LNZ), la minocycline (MNC) ou la moxifloxacine (MOX), dont le choix est dépendant du profil de résistance de chaque isolat clinique.

carbapénèmes présentent des activités différentes sur les deux types de transpeptidases: plus efficaces contre les DDT pour les céphalosporines, supérieures contre les LDT pour les carbapénèmes [16].

Les macrolides représentent une autre classe d'antibiotiques. Ils ciblent, quant à eux, la synthèse protéique en interagissant avec l'ARN ribosomique (ARNr) 235 de la grande sous-unité du ribosome (50S). Ces antibiotiques empêchent ainsi l'allongement de la chaîne peptidique en bloquant le tunnel de sortie par lequel les peptides nouvellement synthétisés s'éloignent du centre peptidyltransférase [17]. Les macrolides, tels que la clarithromycine ou l'azithromycine ont un effet bactériostatique auquel malheureusement M. abscessus subsp. abscessus et M. abscessus subsp. bolletii sont souvent résistantes [18].

Les aminoglycosides sont des molécules hydrophiles qui inhibent la synthèse des protéines en se liant au site $A$ de l'ARNr 16S constituant la petite sous-unité des ribosomes [19]. Cette interaction est à l'origine d'une mauvaise lecture du codon lors de la délivrance de l'ARN de transfert (ARNt) des aminoacyls et, par conséquent, provoquent une traduction erronée de l'ARN messager (ARNm).

Les tétracyclines inhibent également la synthèse protéique des bactéries mais en prévenant l'association des aminoacyl-ARNt aux ribosomes bactériens [20]. Les membres de cette famille, comme la minocycline, ne présentent généralement qu'une activité modeste contre $M$. abscessus. Dérivée de la minocycline, la tigécycline fait exception. Elle est la seule tétracycline à présenter une chaîne 2-tert-butylglycylamino liée au carbone $\mathrm{C}-9$ du cycle aromatique du squelette de la molécule (Figure 3). Ce substituant augmente l'affinité de la molécule pour le ribosome, lui conférant une forte activité inhibitrice ainsi que la capacité d'échapper aux mécanismes de résistance aux tétracyclines les plus couramment développés par les bactéries [21].

Le traitement de l'infection par $M$. abscessus par ces antibiotiques, dont la durée excède souvent 12 mois, s'accompagne néanmoins d'effets secondaires notoires, ce qui conduit, malgré leur efficacité, à de nombreuses impasses thérapeutiques.

\section{La polyrésistance de M. abscessus aux diverses classes d'antibiotiques \\ Une résistance innée de la bactérie}

La paroi mycobactérienne, une barrière naturelle

Si les mécanismes de résistance développés par M. abscessus sont nombreux et variés, la paroi mycobactérienne, unique en son genre par la diversité des lipides qui la constituent, joue le rôle de première barrière à l'encontre de nombreuses molécules exogènes, dont certains antibiotiques. Comme toute cellule, la bactérie est bordée d'une membrane constituée d'une bicouche lipidique: la membrane interne (MI) (Figure 4A). Ancrés dans cette bicouche, se trouvent une grande variété de glycoconjugués: des glycolipides et des lipoglycanes, tels que le phosphatidyl-myo-inositol mannoside, le lipomannane ou le lipoarabinomannane [22] $(\rightarrow)$.

Entourant cette membrane interne,

$(\rightarrow)$ Voir la Synthèse de L. Kremer et al., $m / s n^{\circ} 6-7$, juin-juillet 1999, page 842 l'espace périplasmique est constitué du peptidoglycane, un hétéropolymère lié de manière covalente à une couche d'arabinogalactane, elle-même estérifiée par des acides mycoliques, des acides gras à longues chaînes carbonées. Ces acides mycoliques, caractéristiques des mycobactéries, constituent le feuillet interne de la mycomembrane. Dans le feuillet externe de cette dernière viennent s'insérer des lipides et glycolipides, tels que le tréhalose monomycolate, le tréhalose dimycolate, le tréhalosepolyphléate mais aussi des glycopeptidolipides [23]. Cette vaste panoplie de lipides participe grandement aux propriétés de résistance de la paroi, par exclusion de taille des molécules exogènes, mais aussi par son caractère hydrophobe, conférant une résistance naturelle aux antibiotiques hydrophiles.

L'export actif à travers la membrane

Dans la membrane interne, de nombreuses protéines jouant le rôle de pompes à efflux sont insérées (Figure 4B). Parmi celles-ci, les protéines de la famille MmpL (Mycobacterial membrane protein Large) [24] permettent de maintenir l'homéostasie de la bactérie. Elles sont également responsables d'une balance physiologique permettant l'export de toxines et de métabolites produits par la bactérie. Ces protéines membranaires ont une grande variété de substrats, comme les facteurs de virulence, mais elles ont aussi un rôle indispensable dans le transport de lipides et dans l'élaboration de la paroi bactérienne. Dans certains cas, les 


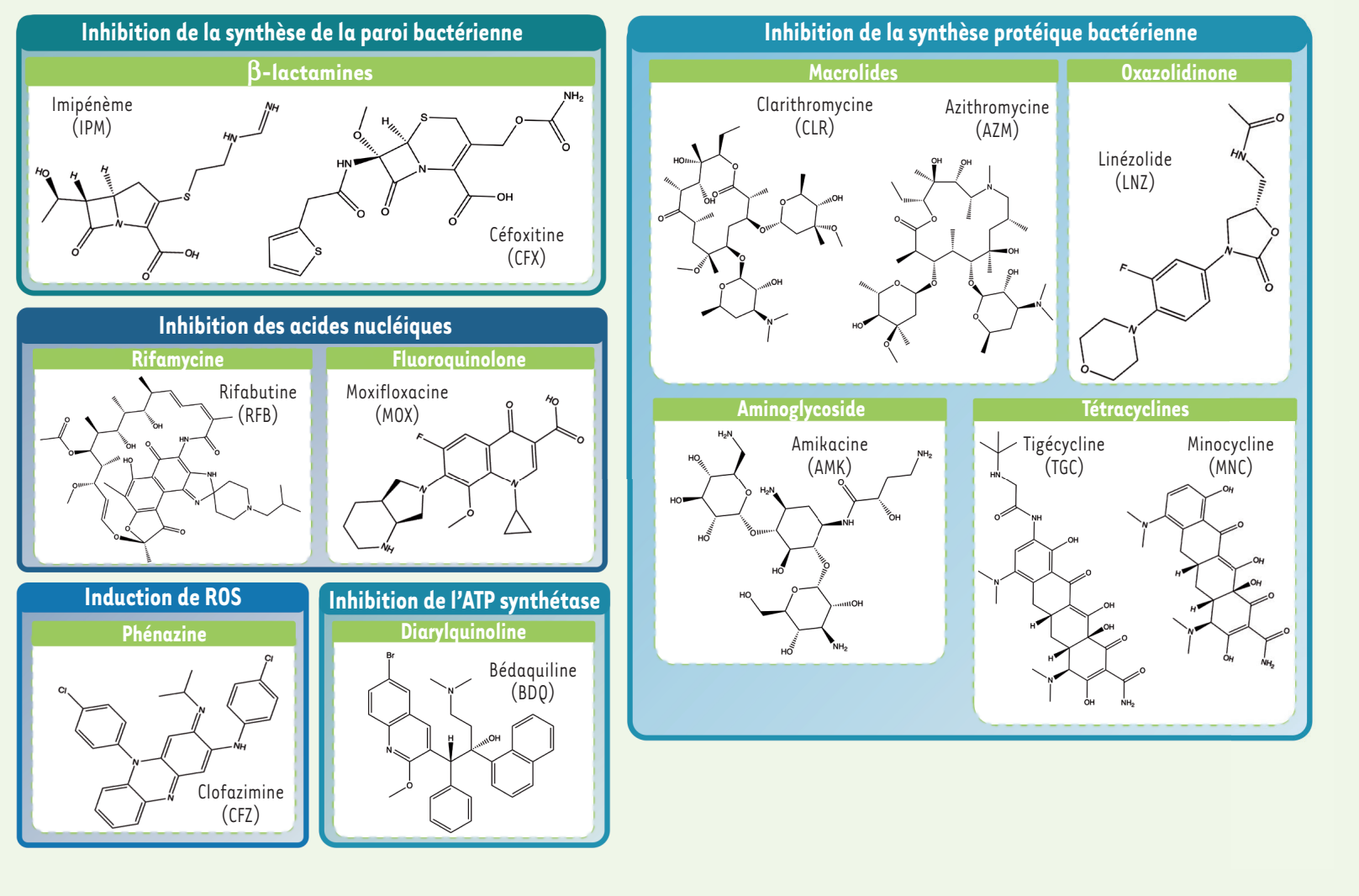

Figure 3. Structure chimique des principaux antibiotiques utilisés dans le traitement des infections pulmonaires à M. abscessus. Les principales classes d'antibiotiques préconisées dans le traitement ainsi que leur mode d'action sont indiqués. ROS : reactive oxygen species.

pompes à efflux peuvent participer à l'export, du cytoplasme vers le milieu extérieur, de substances exogènes (telles que les antibiotiques) qui auraient pénétré dans la cellule. Certaines de ces protéines $\mathrm{MmpL}$ agissent de concert avec des protéines MmpS (Mycobacterial membrane protein Small), dont les gènes sont adjacents à ceux codant les MmpL. Le cluster de gènes MAB_2300/MAB_2301, codant les protéines $\mathrm{MmpS} / \mathrm{MmpL}$, est ainsi impliqué dans l'export d'antibiotiques comme la bédaquiline (BDQ) et la clofazimine (CFZ) (Figure 4B). Leur capacité d'export n'induit toutefois pas de résistances importantes [25] (Tableau I). Une protéine apparentée à la pompe à efflux LmrS (lincomycin resistance protein of Staphylococcus aureus), ainsi que MmpL9, participent à la résistance au linézolide (LNZ) chez M. abscessus. En effet, des niveaux élevés de transcrits du gène codant MmpL9 ont été décrits chez des souches résistantes au linézolide, témoignant de l'implication probable de cette molécule dans l'export de l'antibiotique [26] (Figure 4B). La résistance de M. abscessus aux analogues du thiacétazone (TAC) s'explique également par l'export actif de ces antibiotiques par le système MAB_4383c/MAB_4382c, homologue de $\mathrm{mmpS5/mmpL5}$ chez Mycobacterium tuberculosis [27].

Si l'ensemble de ces transporteurs confèrent une perméabilité ajustable à la paroi bactérienne et représentent ainsi des mécanismes de résistance intrinsèque aux antibiotiques chez $M$. abscessus, nos connaissances sur l'implication d'autres systèmes de transport poten- tiels dans la résistance aux antibiotiques de la bactérie restent encore limitées. Toutefois, diverses études sont actuellement menées dans le but d'identifier des inhibiteurs spécifiques de ces pompes à efflux afin de potentialiser l'efficacité des traitements [28].

Les enzymes modifiant/inactivant les antibiotiques

À côté de ces systèmes d'export, M. abscessus regorge d'une grande variété d'enzymes capables de modifier chimiquement de nombreux antibiotiques, et ainsi de les rendre inopérants. Ces enzymes, qui limitent donc considérablement les options de traitement, constituent un pan non négligeable du «résistome» de M. abscessus (Tableau 1). Parmi celles-là, une seule, ciblant les $\beta$-lactamines, a été identifiée chez $M$. abscessus : la $\beta$-lactamase Bla $_{M A B}$ (Figure $4 C$ ). Codée par le gène $M A B \_2875$, elle hydrolyse le noyau $\beta$-lactame de nombreuses molécules antibiotiques, comme les pénicillines, les carbapénèmes ou les céphalosporines. En clinique, l'utilisation de certains antibiotiques, comme l'imipénème et la céfoxitine, reste néanmoins possible. La $B \mathrm{a}_{\text {MAB }}$ ne les dégradent en effet que très lentement, leur conférant une certaine efficacité [29]. L'avibactam, développé par AstraZeneca, un inhibiteur spéci- 

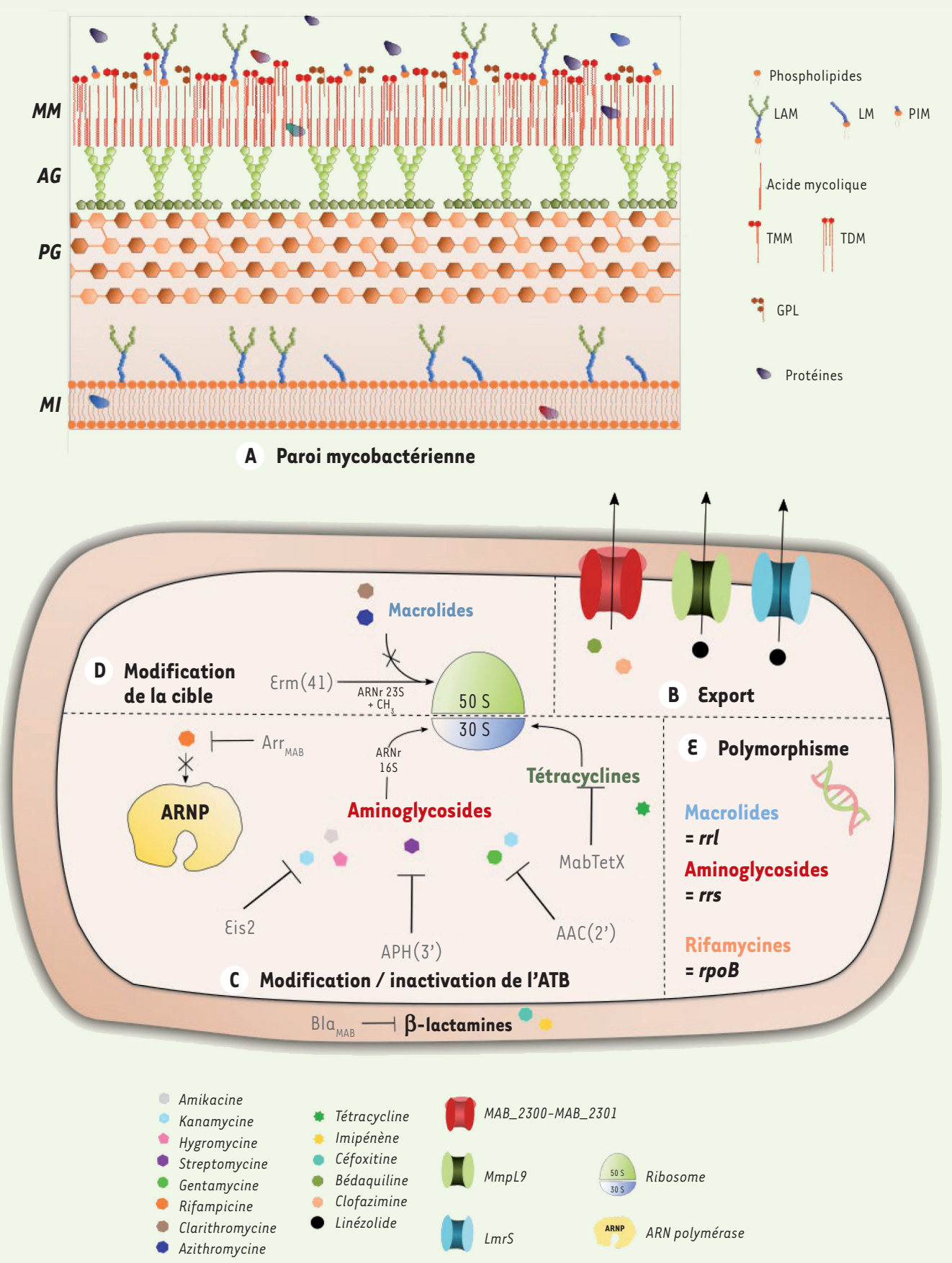

Figure 4. Mécanismes de résistances innée et acquise chez M. abscessus vis-à-vis des principales classes d'antibiotiques. A. La paroi mycobactérienne riche en lipides représente la première ligne de défense contre certains antibiotiques, de par sa forte hydrophobicité et l'exclusion de taille qu'elle provoque. B. L'export d'antibiotiques, tels que la bédaquiline, la clofazimine ou encore le linézolide, implique des protéines transmembranaires, incluant par exemple les transporteurs de type MmpL. C. M. abscessus exprime de nombreuses enzymes capables de modifier et d'inactiver directement les antibiotiques. Arr ${ }_{\text {MAB }}$ (une ADP ribosyltransférase) inhibe l'action de la rifampicine tandis que AAC(2') et Eis2 (des N-acétyltransférases) ainsi que $\mathrm{APH}\left(3^{\prime}\right)$ (une 3'"-0-phosphotransférase) neutralisent l'activité des aminoglycosides. MabTetX (une monooxygénase) inactive l'activité des tétracyclines. D. D'autres enzymes modifient la cible des antibiotiques. La méthyltransférase $\varepsilon r m(41)$ inhibe l'action des macrolides en méthylant l'ARNr $23 S$ de la sous-unité $50 S$ du ribosome, diminuant ainsi l'affinité de l'antibiotique vis-à-vis du ribosome. $\varepsilon$. Le polymorphisme génétique est impliqué dans la résistance à plusieurs classes d'antibiotiques, incluant notamment les macrolides, les aminoglycosides ou les rifamycines. MM : mycomembrane; AG : arabinogalactane; PG : peptidoglycane ; MI : membrane interne ; LAM : lipoarabinomannane ; LM : lipomannane; TMM : tréhalose monomycolate; TDM : tréhalose dimycolate; GPL : glycopeptidolipide ; PIM : phosphatidyl-myo-inositol mannoside. 


\begin{tabular}{|c|c|c|c|}
\hline Antibiotique & Voie métabolique/cible & Gène(s) impliqué(s) & Réf. \\
\hline Isoniazide & $\begin{array}{l}\text { Synthèse des acides mycoliques /énoyl ACP } \\
\text { réductase InhA }\end{array}$ & $\begin{array}{l}\text { Polymorphisme du gène katG codant l'enzyme d'activa- } \\
\text { tion de l'INH }\end{array}$ & [31] \\
\hline Rifampicine & Transcription/ARN polymérase & ADP-ribosylation de la rifampicine par $\mathrm{Arr}_{\mathrm{MAB}}$ & {$[35]$} \\
\hline Aminoglycosides & Synthèse protéique/ARNr $16 \mathrm{~S}$ & $\begin{array}{l}\text { Inactivation par } \mathrm{AAC}\left(2^{\prime}\right) \\
\text { Inactivation par } \mathrm{APH}\left(3^{\prime \prime}\right)\end{array}$ & $\begin{array}{l}{[31]} \\
{[33]}\end{array}$ \\
\hline$\beta$-lactamines & $\begin{array}{l}\text { Synthèse du peptidoglycane/Penicillin- } \\
\text { binding protein }\end{array}$ & Inactivation par Bla ${ }_{M A B}$ & {$[53]$} \\
\hline Tétracycline & $\begin{array}{l}\text { Synthèse protéique/empêche } \\
\text { l'attachement de l'aminoacyl-ARNt au ribo- } \\
\text { some }\end{array}$ & Inactivation par MabTetX & [34] \\
\hline Macrolides & Synthèse protéique/ARNr $23 \mathrm{~S}$ & Modification de la cible par le produit du gène erm(41) & {$[18]$} \\
\hline $\begin{array}{l}\text { Analogues du } \\
\text { thiacétazone }\end{array}$ & $\begin{array}{l}\text { Synthèse des acides mycoliques / déshydratases } \\
\text { HadABC }\end{array}$ & $\begin{array}{l}\text { Pompe à efflux de type } \mathrm{MmpS} / \mathrm{MmpL} \text { codée par } \\
M A B \_4383 c / 4382 c\end{array}$ & {$[54]$} \\
\hline Bédaquiline & Production d'énergie/ATP synthase & $\begin{array}{l}\text { Pompes à efflux de type } \mathrm{MmpS} / \mathrm{MmpL} \text { codées par } \\
M A B \_2300 / 2301 \text { et } M A B \_1135 c / 1134 c\end{array}$ & {$[25,55]$} \\
\hline Clofazimine & $\begin{array}{l}\text { Production de radicaux oxydants produits par } \\
\text { la NADH:quinone oxydoréductase } \\
\text { de type } 2\end{array}$ & $\begin{array}{l}\text { Pompes à efflux de type } \mathrm{MmpS} / \mathrm{MmpL} \text { codées par } \\
\text { MAB_2300/2301 et } M A B \_1135 c / 1134 c\end{array}$ & {$[25,55]$} \\
\hline
\end{tabular}

Tableau I. Résistance innée et acquise aux antibiotiques chez M. abscessus. ACP : acyl carrier protein ; Inh : isoniazid ; KatG : catalase/peroxidase ; MmpL : Mycobacterial membrane protein Large; MmpS : Mycobacterial membrane protein Small.

fique de la $B l a_{M A B}$, permet, également de réduire considérablement la concentration minimale inhibitrice de certaines $\beta$-lactamines. Cette molécule augmente ainsi l'efficacité de ces antibiotiques, ouvrant la voie vers de nouvelles approches thérapeutiques, et donc à l'utilisation de cette famille d'antibiotiques [30].

La résistance de $M$. abscessus aux aminoglycosides s'explique par la présence chez cette bactérie de trois enzymes (deux acétyltransférases et une phosphotransférase) capables de neutraliser cette classe d'antibiotiques. Les deux $\mathrm{N}$-acétyltransférases, $\mathrm{AAC}\left(2^{\prime}\right)$ et $\varepsilon$ is2, sont codées respectivement par les gènes $M A B \_4395$ et MAB_4532c. AAC(2') acétyle le groupement amine situé en position 2' de certains aminoglycosides tels que la kanamycine ou la gentamycine [31], deux antibiotiques qui ne sont pas utilisés dans les traitements cliniques. Eis2, quant à elle, inactive l'amikacine, la kanamycine ou l'hygromycine, en acétylant le premier groupement amine de ces molécules [32]. L'inactivation des gènes codant ces enzymes entraîne une sensibilité accrue des bactéries aux aminoglycosides [31]. La 3"'-0-phosphotransférase $\left(\mathrm{APH}\left(3^{\prime}\right)\right)$, codée par le gène $M A B \_2385$, est, elle, déterminante dans la résistance à la streptomycine [33]. Les modifications chimiques que ces enzymes produisent sur les aminoglycosides, diminuent leur affi- nité pour l'ARNr 165 de la sous-unité 30 S du ribosome, ce qui les rend inopérantes (Figure $4 C$ ).

Bien qu'efficaces contre M. tuberculosis, les tétracyclines n'ont que peu d'effet sur M. abscessus. En effet, M. abscessus exprime une monooxygénase, la MabTetX [34], qui inactive les tétracyclines (Figure 4C). La délétion du gène $M A B_{-}$1496c qui code cette enzyme, entraîne chez la souche mutée, une sensibilité accrue à ce type d'antibiotique, vingt fois plus élevée par rapport à la souche parentale, confirmant l'importance de cette enzyme dans la résistance aux tétracyclines.

La rifampicine est une molécule utilisée en première intention pour le traitement de la tuberculose. M. abscessus reste cependant insensible à l'action de cet antibiotique. La raison de cette résistance repose sur la présence chez $M$. abscessus d'une ribosyltransférase, l'ADP ribosyltransférase Arr $_{M A B}$, codée par le gène MAB_0591. Cette enzyme est capable d'ADP ribosyler la rifampicine, l'empêchant d'agir au niveau de la sousunité $\beta$ de l'ARN polymérase [35] (Figure 4C). La décou- 
verte de dérivés de la rifamycine, plus réfractaires à l'ADP-ribosylation par $\operatorname{Arr}_{M A B}$, représente néanmoins une piste de recherche prometteuse dans la lutte contre M. abscessus [36].

Les enzymes protégeant la cible des antibiotiques

M. abscessus possède également une enzyme capable de protéger les protéines ciblées par les macrolides (Tableau I). La méthyltransférase $\operatorname{Erm}(41)$, codée par le gène $M A B \_2997$, est en effet capable de méthyler l'adénine en position 2058 de l'ARNr 235 de la sous-unité $50 S$ du ribosome [18] (Figure 4D). Par cette méthylation, cette enzyme produit un encombrement stérique qui réduit l'affinité des macrolides pour le tunnel de sortie du polypeptide. Il existe un polymorphisme du gène erm(41) codant cette méthyltransférase, sur le nucléotide en position 28 : thymine ou cytosine. Selon ce polymorphisme, les souches cliniques de M. abscessus présentent une résistance à la clarithromycine ou à l'azithromycine : le sequevar (ou variant) T28 (correspondant à un résidu tryptophane [Trp] en position 10 de la protéine) est en effet associé à une résistance inductible, alors que le sequevar C28 (correspondant à un résidu arginine $[\operatorname{Arg} 10]$ ) est associé à une sensibilité de la bactérie aux macrolides [37]. Le niveau de résistance de la bactérie, qui augmente progressivement en fonction du temps d'exposition à la clarithromycine ou à l'azithromycine, est inductible et directement lié à l'activateur de transcription WhiB7 [38]. Sachant que les macrolides représentent la pierre angulaire de la thérapie antiM. abscessus, cette résistance aux macrolides inductible, observée dans 40 à $60 \%$ des souches cliniques [37], rend compte du problème majeur de santé publique que représente l'infection par cette bactérie.

\section{Polymorphisme nucléotidique et résistance acquise}

Aux mécanismes de résistance innée que nous avons décrits, s'ajoutent des résistances acquises par $M$. abscessus. Ces résistances, fruit de modifications génétiques qui sont retrouvées dans les isolats cliniques, sont le résultat d'une exposition prolongée de la bactérie aux antibiotiques. Des mutations spontanées surviennent ainsi au sein des gènes codant les cibles protéiques des antibiotiques (Tableau / et Figure 4ع), mais d'autres mécanismes additionnels, tels que le transfert génétique entre espèces bactériennes, sont probablement impliqués.

\section{Résistance acquise aux macrolides}

Des mutations du gène $r r l$, qui code l'ARNr 23S [39], touchant les adénines en position 2058 et 2059 de l'ARNr 23S, sont induites au décours des expositions aux antibiotiques. Celles-ci réduisent l'activité des macrolides, et sont retrouvées dans les isolats de M. abscessus, comme c'est le cas pour d'autres espèces mycobactériennes, telles que $M$. avium [39].

\section{Résistance acquise aux aminoglycosides}

Des mutations du gène rrs, qui code l'ARNr $16 \mathrm{~S}$ et qui induisent une résistance aux aminoglycosides, ont été décrites. Quatre mutations, affectant I'ARNr 16S (T1406A, A1408G, C1409T et G1491T) ${ }^{1}$, ont été

\footnotetext{
1 Thymine 1406 > adénine; adénine 1408 > guanine ; cytosine 1409 > thymine ; guanine 1491 > thymine.
}

associées à de hauts niveaux de résistance de $M$. abscessus aux aminoglycosides [40]. Deux nouvelles mutations (C1496T et T1498A)² ont été identifiées récemment [41].

\section{Résistance acquise aux rifamycines}

M. abscessus est réfractaire à l'action de la rifampicine qui est inactivée par l'action de la Arr $_{M A B}$. Toutefois, la rifabutine, de la famille des rifamycines, fait exception. Dans un modèle d'infection chez le poisson-zèbre (zebrafish) $[42,43](\rightarrow)$ ainsi que dans un modèle murin d'infection par M. abscessus [44], cet antibiotique présente des effets

$(\rightarrow)$ Voir la Synthèse de A. Bernut et al., $m / s n^{\circ} 6-7$, juin-juillet 2015, page 638 bactéricides. Ces données confirment la capacité de la rifabutine à inhiber la transcription chez M. abscessus, à l'instar de la rifampicine. Des mutations du gène rpo $B$ qui code la sous-unité $\beta$ de l'ARN polymérase (H447Y, H447D et S452L) ${ }^{3}$ sont cependant à l'origine de la résistance de la bactérie à la rifabutine [43].

\section{Conclusion et perspectives}

Les nombreux mécanismes de résistance de $M$. abscessus, conjugués à l'émergence de souches multirésistantes, limitent considérablement les outils mis à la disposition du clinicien pour traiter les infections pulmonaires causées par cette mycobactérie atypique. Le traitement actuel recommandé ne permet pas, dans la plupart des cas, d'éradiquer des infections à $M$. abscessus. Face à ce problème de santé publique en progression constante, des efforts doivent être faits pour encourager et favoriser des études ayant pour but d'identifier de nouvelles pistes thérapeutiques pour lutter contre les infections persistantes à M. abscessus, avec en particulier, l'identification de nouvelles cibles d'intérêt thérapeutique et le développement de molécules auxquelles cette bactérie serait sensible. Depuis plusieurs années, la recherche se concentre sur la découverte et le repositionnement de molécules actives vis-à-vis de M. abscessus comme, par exemple, la rifabutine $[43,44]$, la bédaquiline $[45,46]$, le tédizolide [47] ou les inhibiteurs du transporteur des acides mycoliques MmpL3 [48, 49] $(\rightarrow)$. D'autres molécules ou formulations sont actuellement testées dans des essais cliniques, comme l'inhalation d'amikacine liposo-

$(\rightarrow)$ Voir la Nouvelle

de C. Raynaud et L. Kremer, $m / s n^{\circ} 8-9$, août-septembre 2020, page 691 male ou le monoxyde d'azote [50]. Des approches

2 Cytosine $1496>$ Thymine ; thymine $1498>$ adénine.

${ }^{3}$ Ou c.1339C >T ; c.1339C>G ; c. 1355C>T 
alternatives à l'antibiothérapie sont également en cours d'étude. La phagothérapie, par exemple, très utilisée au début $\mathrm{du} x \mathrm{x}^{\mathrm{e}}$ siècle, est remise au devant de la scène dans la lutte contre les infections par les MNT [51] $(\rightarrow)$.

Ainsi, l'administration d'un cocktail de mycobactériophages spécifiques à une souche multirésistante de $M$. abscessus, dans le cadre du traitement d'une infection disséminée chez une jeune patiente atteinte de mucoviscidose, a amélioré l'état clinique de la patiente et a suscité beaucoup d'espoir [52]. Si la phagothérapie reste encore anecdotique dans les pays occidentaux, elle pourrait néanmoins permettre d'établir des traitements personnalisés avec une toxicité moindre, notamment chez les patients particulièrement réfractaires à l'antibiothérapie classique. $\diamond$

\section{SUMMARY}

Mycobacterium abscessus, a model of resistance to multiple antibiotic classes

Mycobacterium abscessus is an environmental fast-growing, nontuberculous mycobacterium responsible for severe lung infections, especially in patients with underlying lung disorders such as cystic fibrosis. The standard chemotherapy combines a $\beta$-lactam (imipenem or cefoxitin), an aminoglycoside (amikacin) and a macrolide (clarithromycin or azithromycin). However, resistance of this bacterium to most antibiotic classes, including nearly all anti-tubercular drugs, leads frequently to treatment failure and considerably reduces the therapeutic arsenal available to the clinician. A comprehensive understanding of the innate and acquired resistance mechanisms is thus necessary to counteract $M$. abscessus lung infections. $\diamond$

\section{REMERCIEMENTS}

Morgane Illouz est bénéficiaire d'une bourse de thèse de l'Association Grégory Lemarchal et de Vaincre la Mucoviscidose (RF20200502678). Matthéo Alcaraz bénéficie d'une bourse de thèse du Ministère de l'Enseignement Supérieur, de la Recherche et de l'Innovation.

\section{LIENS D'INTÉRÊT}

Les auteurs déclarent n'avoir aucun lien d'intérêt concernant les données publiées dans cet article.

\section{RéFÉRENCES}

1. Moore M, Frerichs JB. An unusual acid-fast infection of the knee with subcutaneous, abscess-like lesions of the gluteal region. J Invest Dermatol 1953; 20 : 133-69.

2. Minias A. Subspecies-specific sequence detection for differentiation of Mycobacterium abscessus complex. Sci Rep 2020 ; 10: 16415

3. Bryant JM, Grogono DM, Rodriguez-Rincon D, et al. Emergence and spread of a humantransmissible multidrug-resistant nontuberculous mycobacterium. Science 2016 ; $354: 751-7$.

4. Johansen MD, Herrmann J-L, Kremer L. Non-tuberculous mycobacteria and the rise of Mycobacterium abscessus. Nat Rev Microbiol 2020 ; 18 : 392-407.

5. Kwak N, Dalcolmo MP, Daley CL, et al. Mycobacterium abscessus pulmonary disease: individual patient data meta-analysis. Eur Respir J 2019 ; $54: 1801991$.

6. Choo SW, Wee WY, Ngeow YF, et al. Genomic reconnaissance of clinical isolates of emerging human pathogen Mycobacterium abscessus reveals high evolutionary potential. Sci Rep ; 4: 4061.

7. Ripoll F, Pasek S, Schenowitz C, et al. Non mycobacterial virulence genes in the genome of the emerging pathogen Mycobacterium abscessus. PloS One $2009 ; 4$ : e5660.

8. Férec C. La mucoviscidose : du gène à la thérapeutique. Med Sci (Paris) 2021 ; 37 : 618-24.

9. Davies JC, Alton EWFW, Bush A. Cystic fibrosis. BMJ 2007 ; 335 : 1255-9.
10. Catherinot $\varepsilon$, Roux AL, Macheras $\varepsilon$, et al. Acute respiratory failure involving an R variant of Mycobacterium abscessus. J Clin Microbiol 2009 ; 47 : 271-4.

11. Bernut A, Dupont C, Ogryzko NV, et al. CFTR Protects against Mycobacterium abscessus infection by fine-tuning host oxidative defenses. Cell Rep. 2019; $26: 1828-40$.

12. Nessar R, Cambau $\varepsilon$, Reyrat JM, et al. Mycobacterium abscessus: a new antibiotic nightmare. J Antimicrob Chemother 2012 ; $67: 810-18$.

13. Yam YK, Alvarez N, Go ML, et al. Extreme drug tolerance of Mycobacterium abscessus persisters. Front Microbiol $2020 ; 11: 359$.

14. Haworth CS, Banks J, Capstick T, et al. British thoracic society guidelines for the management of non-tuberculous mycobacterial pulmonary disease (NTM-PD). Thorax 2017 ; 72 : iil-64.

15. Lavollay M, Fourgeaud M, Herrmann JL, et al. The peptidoglycan of Mycobacterium abscessus is predominantly cross-linked by L,Dtranspeptidases. J Bacteriol 2011 ; 193 : 778-82.

16. Dubée V, Triboulet S, Mainardi JL, et al. Inactivation of Mycobacterium tuberculosis L,D-transpeptidase LdtMtl by carbapenems and cephalosporins. Antimicrob Agents Chemother 2012 ; 56 : 4189-95.

17. Wilson DN. Ribosome-targeting antibiotics and mechanisms of bacterial resistance. Nat Rev Microbiol $2014 ; 12$ : 35-48.

18. Nash KA, Brown-Elliott BA, Wallace RJ. A novel gene, erm(41), confers inducible macrolide resistance to clinical isolates of Mycobacterium abscessus but is absent from Mycobacterium chelonae. Antimicrob Agents Chemother $2009 ; 53$ : 1367-76.

19. Kotra LP, Haddad J, Mobashery S. Aminoglycosides: perspectives on mechanisms of action and resistance and strategies to counter resistance. Antimicrob Agents Chemother 2000 ; $44: 3249-56$.

20. Chopra I, Roberts M. Tetracycline antibiotics: Mode of action, applications, molecular biology, and epidemiology of bacterial resistance. Microbiol Mol Biol Rev 2001 ; 65 : 232-60.

21. Schedlbauer A, Kaminishi T, Ochoa-Lizarralde B, et al. Structural characterization of an alternative mode of tigecycline binding to the bacterial ribosome. Antimicrob Agents Chemother 2015 ; 59 : 2849-54.

22. Kremer L, Besra G, Brennan P, et al. Le lipoarabinomannane : structure et fonctions d'un glycolipide impliqué dans la pathogénie tuberculeuse. Med Sci $1999 ; 15: 842-50$.

23. Brennan PJ, Nikaido H. The envelope of mycobacteria. Annu Rev Biochem $1995 ; 4: 29-63$.

24. Viljoen A, Dubois V, Girard-Misguich F, et al. The diverse family of $\mathrm{MmpL}$ transporters in mycobacteria: from regulation to antimicrobial developments: MmpL structure and diversity in mycobacteria. Mol Microbiol $2017 ; 104: 889-904$

25. Richard M, Gutiérrez AV, Viljoen A, et al. Mutations in the MAB_2299c TetR regulator confer cross-resistance to clofazimine and bedaquiline in Mycobacterium abscessus. Antimicrob Agents Chemother 2019; 63 : e01316-18.

26. Ye M, Xu L, Zou Y, et al. Molecular analysis of linezolid-resistant clinical isolates of Mycobacterium abscessus. Antimicrob Agents Chemother 2018 ; $63: e 01842-18$

27. Halloum I, Viljoen A, Khanna V, et al. Resistance to thiacetazone derivatives active against Mycobacterium abscessus involves mutations in the MmpL5 transcriptional repressor MAB_4384. Antimicrob Agents Chemother 2017 ; 61: e02509-16.

28. Vianna JS, Ramis IB, Bierhals D, et al. Tetrahydropyridine derivative as efflux inhibitor in Mycobacterium abscessus. J Glob Antimicrob Resist 2019; 17 : 296-9.

29. Soroka D, Dubee V, Soulier-Escrihuela 0 , et al. Characterization of broadspectrum Mycobacterium abscessus class A- $\beta$ lactamase. J Antimicrob Chemother $2014 ; 69: 691-6$.

30. Dubee V, Bernut A, Cortes $M$, et al. $\beta$-Lactamase inhibition by avibactam in Mycobacterium abscessus. J Antimicrob Chemother $2015 ; 70: 1051-8$.

31. Rominski A, Selchow P, Becker K, et al. Elucidation of Mycobacterium abscessus aminoglycoside and capreomycin resistance by targeted deletion of three putative resistance genes. J Antimicrob Chemother 2017 ; 72 : 21912200.

32. Ung KL, Alsarraf HMAB, Olieric V, et al. Crystal structure of the aminoglycosides $\mathrm{N}$-acetyltransferase Eis2 from Mycobacterium abscessus. FEBS J 2019 ; 286 : 4342-55.

33. Dal Molin M, Gut M, Rominski A, et al. Molecular mechanisms of intrinsic streptomycin resistance in Mycobacterium abscessus. Antimicrob Agents Chemother 2017 ; 62 : e01427-17. 


\section{RÉFÉRENCES}

34. Rudra $P$, Hurst-Hess K, Lappierre $P$, et al. High levels of intrinsic tetracycline resistance in Mycobacterium abscessus are conferred by a tetracycline-modifying monooxygenase. Antimicrob Agents Chemother 2018 ; 62 : e00119-18.

35. Rominski A, Roditscheff A, Selchow P, et al. Intrinsic rifamycin resistance of Mycobacterium abscessus is mediated by ADP-ribosyltransferase MAB_0591. J Antimicrob Chemother 2017; 72 : 376-84.

36. Combrink KD, Ramos AR, Spring S, et al. Rifamycin derivatives active against pathogenic rapidlygrowing mycobacteria. Bioorg Med Chem Lett $2019 ; 29: 2112-5$.

37. Bastian $S$, Veziris N, Roux A-L, et al. Assessment of clarithromycin susceptibility in strains belonging to the Mycobacterium abscessus group by erm (41) and rrl sequencing. Antimicrob Agents Chemother $2011 ; 55: 775-81$.

38. Richard M, Gutiérrez AV, Kremer L. Dissecting erm(41)-mediated macrolide-inducible resistance in Mycobacterium abscessus. Antimicrob Agents Chemother 2020 ; 64 : e01879-19.

39. Wallace RJ, Meier A, Brown BA, et al. Genetic basis for clarithromycin resistance among isolates of Mycobacterium chelonae and Mycobacterium abscessus. Antimicrob Agents Chemother 1996; 40 1676-81.

40. Nessar R, Reyrat JM, Murray A, et al. Genetic analysis of new 165 rRNA mutations conferring aminoglycoside resistance in Mycobacterium abscessus. J Antimicrob Chemother 2011; 66 : 1719-24

41. Kim Sy, Kim DH, Moon SM, et al. Association between 16S rRNA gene mutations and susceptibility to amikacin in Mycobacterium avium complex and Mycobacterium abscessus clinical isolates. Sc Rep $2021 ; 11: 6108$.

42. Bernut $A$, Lutfalla $G$, Kremer $L$. Regard à travers le danio pour mieux comprendre les interactions hôte/pathogène. Med Sci (Paris) 2015 ; $31: 638$-46.

43. Johansen MD, Daher W, Roquet-Banères $F$, et al. Rifabutin is bactericidal against intracellular and extracellular forms of Mycobacterium abscessus. Antimicrob Agents Chemother 2020;64: e00363-20.

44. Dick T, Shin SJ, Koh WJ, et al. Rifabutin is active against Mycobacterium abscessus in mice. Antimicrob Agents Chemother 2019 ; 64 : e01943-19.

45. Philley JV, Wallace RJ, Benwill JL, et al. Preliminary results of bedaquiline as salvage therapy for patients with nontuberculous mycobacterial lung disease. Chest $2015 ; 148: 499-506$.
46. Dupont C, Viljoen A, Thomas S, et al. Bedaquiline inhibits the ATP synthase in Mycobacterium abscessus and is effective in infected zebrafish. Antimicrob Agents Chemother 2017 ; 61 : e01225-17.

47. Le Run $\varepsilon$, Arthur M, Mainardi J-L. In vitro and intracellular activity of imipenem combined with tedizolid, rifabutin, and avibactam against Mycobacterium abscessus. Antimicrob Agents Chemother 2019 ; 63 : e01915-8.

48. Dupont C, Viljoen A, Dubar F, et al. A new piperidinol derivative targeting mycolic acid transport in Mycobacterium abscessus: Inhibiting mycolic acid transport in M. abscessus. Mol Microbiol 2016; 101 : 515-29.

49. Raynaud C, Kremer L. Vers un nouvel espoir pour traiter les infections persistantes à Mycobacterium abscessus ? Med Sci (Paris) 2020 ; $36: 691-4$

50. Wu ML, Aziz DB, Dartois V, et al. NTM drug discovery: status, gaps and the way forward. Drug Discov Today 2018 ; 23 : 1502-19.

51. Jordan B. Cent ans après, le retour de la phagothérapie? Med Sci (Paris) $2019 ; 35: 806-9$.

52. Dedrick RM, Guerrero-Bustamante CA, Garlena RA, et al. Engineered bacteriophages for treatment of a patient with a disseminated drugresistant Mycobacterium abscessus. Nat Med $2019 ; 25: 730-3$.

53. Dubée $V$, Soroka $D$, Cortes $M$, et al. Impact of $\beta$-lactamase inhibition on the activity of ceftaroline against Mycobacterium tuberculosis and Mycobacterium abscessus. Antimicrob. Agents Chemother 2015 ; 59 : 2938-41.

54. Richard M, Gutiérrez AV, Viljoen AJ, et al. Mechanistic and structural insights into the unique TetR-dependent regulation of a drug efflux pump in Mycobacterium abscessus. Front Microbiol $2018 ; 9$ : 649

55. Gutiérrez AV, Richard M, Roquet-Banères $F$, et al. The TetR family transcription factor MAB_2299c regulates the expression of two distinct MmpS-MmpL efflux pumps involved in cross-resistance to clofazimine and bedaquiline in Mycobacterium abscessus. Antimicrob Agents Chemother $2019 ; 63$ : e01000-19.

\section{TIRÉS À PART}

L. Kremer

P ossédées du Malin au Moyen-Âge, les sorcières hystériques sont vouées au bûcher. Enfermées au xVII siècle, maltraitées, elles rejoignent la Cour des Miracles de l'Hospice de la Vieillesse-Femmes à la Salpêtrière... Jusqu'à ce que le Dr JeanMartin Charcot (1825-1893) mène le combat qui transforme l'ancien hospice en hôpital : l'École de la Salpêtrière de

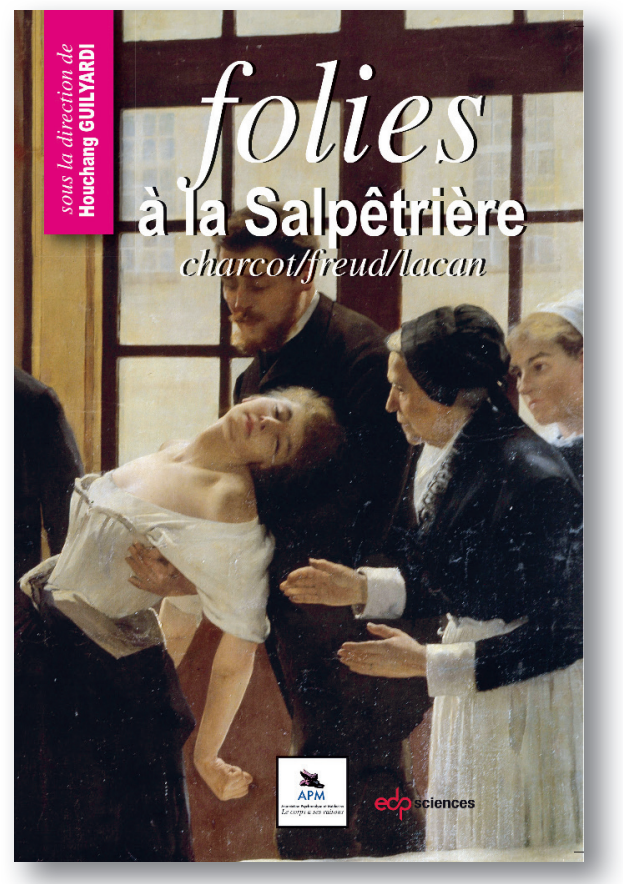

ISBN : 978-2-7598-1268-4
240 pages Paris est née, qui devient lieu de recherche, d'enseignement et de soins, de renommée internationale.

Jean Martin Charcot n'a pas bonne presse, et pourtant... Hystérie et folie traversent les siècles, prenant les formes de «l'air du temps ».

De l'utérus migrateur d'Hippocrate aux recherches neurologiques de Charcot. Du désir inconscient avec Freud à la jouissance du parlêtre chez Lacan... C'est à cette traversée historique et conceptuelle que nous convie cet ouvrage. 